\title{
LncRNA H19 regulates ID2 expression through competitive binding to hsa-miR-19a/b in acute myelocytic leukemia
}

\author{
TONG-FENG ZHAO ${ }^{1}$, HUI-ZHEN JIA ${ }^{1}$, ZHEN-ZHEN ZHANG ${ }^{1}$, XIAO-SU ZHAO ${ }^{2,3}$, \\ YAN-FEN ZOU ${ }^{4}$, WEI ZHANG ${ }^{5}, \mathrm{JUN} \mathrm{WAN}^{1^{*}}$ and XIAO-FAN CHEN ${ }^{5^{*}}$ \\ ${ }^{1}$ Shenzhen Key Laboratory for Neuronal Structural Biology, Biomedical Research Institute, \\ Shenzhen Peking University-The Hong Kong University of Science and Technology Medical Center, \\ Shenzhen, Guangdong 518036; ${ }^{2}$ Department of Hematology, Peking University People's Hospital, Peking University Institute \\ of Hematology, Beijing Key Laboratory of Hematopoietic Stem Cell Transplantation; ${ }^{3}$ Collaborative Innovation Center of \\ Hematology, Peking University, Beijing 100044; ${ }^{4}$ Department of Dermatology, Peking University \\ Shenzhen Hospital; ${ }^{5}$ Shenzhen Key Laboratory for Translational Medicine of Dermatology, \\ Biomedical Research Institute, Shenzhen Peking University-The Hong Kong University of \\ Science and Technology Medical Center, Shenzhen, Guangdong 518036, P.R. China
}

Received December 19, 2016; Accepted July 6, 2017

DOI: $10.3892 / \mathrm{mmr} .2017 .7029$

\begin{abstract}
Acute myelocytic leukemia (AML) is the most common type of acute leukemia. Long non-coding RNAs (lncRNAs) serve an important role in regulating gene expression through chromatin modification, transcription and post-transcriptional processing. LncRNA H19 was considered as an independent prognostic marker for patients with tumors. The expression of IncRNA H19 was identified to be significantly upregulated in bone marrow samples from patients with AML-M2. Furthermore, it was demonstrated that the knockdown of lncRNA H19 resulted in increased expression of hsa-microRNA (miR)-19a/b and decreased expression of inhibitor of DNA binding 2 (ID2) in AML cells. The knockdown of lncRNA H19 inhibited the proliferation of AML cells in vitro, which could be partially reversed by ID2 overexpression. Furthermore, the results of the bioinformatic analysis revealed potential hsa-miR-19a/b-3p binding sites in lncRNA
\end{abstract}

Correspondence to: Dr Xiao-Fan Chen, Shenzhen Key Laboratory for Translational Medicine of Dermatology, Biomedical Research Institute, Shenzhen Peking University-The Hong Kong University of Science and Technology Medical Center, 1120 Lianhua Road, Shenzhen, Guangdong 518036, P.R. China

E-mail: littlecanva@163.com

Dr Jun Wan, Shenzhen Key Laboratory for Neuronal Structural Biology, Biomedical Research Institute, Shenzhen Peking University-The Hong Kong University of Science and Technology Medical Center, 1120 Lianhua Road, Shenzhen, Guangdong 518036, P.R. China

E-mail:wanj@ust.hk

*Contributed equally

Key words: long non-coding RNA, acute myelocytic leukemia, LncRNA H19, ID2, cell proliferation
H19 and ID2. Altogether, the results of the present study suggest that lncRNA H19 regulates the expression of ID2 through competitive binding to hsa-miR-19a and hsa-miR-19b, which may serve a role in AML cell proliferation.

\section{Introduction}

Acute myelocytic leukemia (AML), a heterogeneous clonal disorder of hematopoietic cells, is the most common acute leukemia in adults (1). AML was characterized as an abnormal proliferation of myeloid progenitor cells and an arrest in cellular differentiation. According to the French-American-British classification system, AML was classified into eight major AML subtypes (FAB, M0-M7). Despite great progress achieved in this field, the etiology and pathogenesis of AML is still not fully understood.

In the past, non-coding RNA (ncRNA) had been considered as transcriptional noise. Recently, it was suggested that ncRNAs participated in a wide range of processes including protein translation, mRNA splicing, chromatin organization, and the regulation of gene expression. According to their size, ncRNAs are generally classified into small ncRNAs (sncRNAs; <200 nucleotides, including microRNAs, Piwi-interacting RNAs and small nucleolar RNAs) and long ncRNAs (IncRNAs, $>200$ nucleotides) $(2,3)$. The pivotal regulatory role of ncRNAs in the development of solid tumors and hematological malignancy has attracted increasing attention. LncRNAs are often expressed in a temporally and spatially specific manner $(4,5)$, which also play a critical role in cancer development $(6,7)$.

LncRNA H19 is one of the first discovered lncRNAs. LncRNA H19 is highly expressed during embryonic and fetus stages, while it is downregulated in most of tissues shortly after birth (8). It was found that the silence of lncRNA H19 inhibited tumor cell proliferation, invasion and proliferation, while the overexpression of lncRNA H19 conferred growth 
advantage to tumor cell $(9,10)$. Moreover, it was suggested that lncRNA H19 expression was negatively related to survival rate of tumor patients $(11,12)$. Level of lncRNA H19 in plasma was stable in gastric cancer, which could be used as an biomarker of cancer diagnosis, especially for early cancer screening and dynamic monitoring of tumor after surgery $(13,14)$. Zhang et al have proved that the level of lncRNA H19 overexpression was positively related to Tumor-Node-Metastasis (TNM) stage and tumor size in non-small cell lung cancer, which could be also used as an independent prognostic factor of overall survival of patients (15). However, there is little research about the expression of lncRNA H19 in AML patients, and the role of lncRNA H19 in AML pathogenesis is still unknown.

In this study, it was showed that the expression of IncRNA H19 was significantly upregulated in bone marrow samples from AML-M2 patients. Knockdown of 1ncRNA H19 inhibited AML cell proliferation, which gave rise to the upregulated expression of hsa-miR-19a/b and the downregulated expression of inhibitor of DNA binding 2 (ID2), one of the target genes of hsa-miR-19a/b.

\section{Materials and methods}

Cell culture and transfection. HL-60 cells, KG-1a cells and HEK-293T cells were cultured in RPMI-1640 medium (Gibco, Grand Island, NY, USA) supplied with $10 \%$ fetal bovine serum, $100 \mathrm{U} / \mathrm{ml}$ penicillin, and $100 \mu \mathrm{g} / \mathrm{ml}$ streptomycin, at $37^{\circ} \mathrm{C}$ in a $5 \% \mathrm{CO}_{2}$ atmosphere. Has-miR-19a/b mimic, Has-miR-19a/b inhibitor and their respective scramble controls were obtained from Guangzhou RiboBio Co., Ltd. (Guangzhou, China). LncRNA H19 siRNA was purchased from Invitrogen Life Technologies (Waltham, MA, USA). The sequences of mimics and siRNAs were listed in Table I.

Transfection of HL-60 cells and KG-1a cells were electroporated with Neon ${ }^{\circledR}$ Transfection System (Invitrogen Life Technologies), and transferred to RPMI-1640 medium supplied with $10 \%$ fetal bovine serum. Cell viability of the electroporated cells was more than 70\%. HEK-293T Cells were transfected with Lipofectamine ${ }^{\circledR} 2000$ (Invitrogen Life Technologies) according to the the manufacturer's protocol.

Cell proliferation assay. Cells were seeded in a 96-well plate and cultured in normal medium. At $0,24,48$ and $72 \mathrm{~h}$ after transfection, the cells were incubated in $0.1 \mathrm{mg} / \mathrm{ml} \mathrm{MTT} \mathrm{at}$ $37^{\circ} \mathrm{C}$ for $4 \mathrm{~h}$ and lysed in dimethylsulphoxide (DMSO) at room temperature for $10 \mathrm{~min}$. The absorbance in each well was measured by a microplate reader (Bio-Rad, Hercules, CA, USA). Data are the mean \pm standard deviation of three independent experiments.

Soft agar colony formation assay. A 2-ml layer of $0.7 \%$ agar in RPMI-1640 medium with 10\% FBS was poured into 6-well plates. Cells were resuspended in $0.3 \%$ agar in RPMI-1640 medium with $10 \% \mathrm{FBS}$, and $1 \mathrm{ml}$ cell suspension was poured on top of the base layer. The suspension was allowed to solidify and incubated at $37^{\circ} \mathrm{C}$ in $5 \% \mathrm{CO}_{2}$ for 14 days. The experiment was performed in triplicate and repeated three times.

Patients and tissue collection. Bone marrow samples were obtained from 107 patients with AML (58 female/49 male, mean age: $40.14 \pm 17.94$ years) and 53 matched healthy subjects (26 female/27 male, mean age: $40.89 \pm 11.75$ years). The 107 patients included 46 AML patients with maturation (AML-M2), 20 AML patients with AML-M3 (acute promyelocytic leukemia, APL) and 41 AML patients with other types of AML. The diagnosis of leukemia was made by morphologic and cytochemical studies of bone marrow smears. All patients were not taking any anti-leukemic therapy at the moment of blood sampling and were newly diagnosed with AML. The study was approved by the Ethics Review Committee for Human Studies of the Peking University Health Science Center (Beijing, China). All participants provided written informed consent before any blood sampling.

Plasmid construction. In order to generate a reporter construct, the $3^{\prime}$ untranslated region (3'UTR) of the ID2 gene was cloned into the psiCHECK-2 luciferase vector (Promega Corp., Madison, WI, USA). In order to generate ID2 expression construct, the coding sequence (CDS) of the ID2 gene were cloned into pcDNA4 (Addgene Inc., Cambridge, MA, USA). The sequences of primers were listed in Table I.

RNA extraction and quantitative polymerase chain reaction. RNA was extracted from bone marrow samples and AML cells using TRIzol (Invitrogen Life Technologies) following the manufacturer's protocol. $1 \mu \mathrm{g}$ RNA was reverse transcribed into complementary DNA (cDNA) using the GoScript ${ }^{\mathrm{TM}}$ Reverse Transcription System (Promega Corp.) following the manufacturer's instructions. The ABI StepOne Plus (Applied Biosystems, Foster City, CA, USA) was used to perform the amplification reaction.

Primers for amplification of lncRNA H19, ID2, and GAPDH were synthesized by Invitrogen Life Technologies. qPCR was performed using 96 well plates (Bio-Rad) in $20 \mu \mathrm{l}$ mixture containing $10 \mu \mathrm{l}$ SYBR-Green qPCR Master Mix (Bio-Rad), 10 pmol forward primers and reverse primers, and the appropriate amount of cDNA. The samples were denatured at $95^{\circ} \mathrm{C}$ for $10 \mathrm{~min}$, and then amplified during 40 cycles at $95^{\circ} \mathrm{C}$ for $30 \mathrm{sec}, 60^{\circ} \mathrm{C}$ for $30 \mathrm{sec}$, and $72^{\circ} \mathrm{C}$ for $30 \mathrm{sec}$ on an iQ5 Real-Time thermal cycler (Bio-Rad). Each sample was assayed in triplicate, and $\mathrm{C}_{\text {quantification }}$ cycle $(\mathrm{Cq})$ values were measured. A mean $\mathrm{Cq}$ value for each triplicate measurement was calculated. Relative gene expression was then calculated using $2^{-\Delta \Delta C q}$ method. The sequences of primers were also listed in Table I.

Western blot analysis. Proteins were extracted from cells with RIPA lysis buffer (KenGen Biotech, Nanjing, China) and were quantified using a BCA Protein Assay kit (Thermo Fisher Scientific, Inc., Waltham, MA, USA). A total of $30 \mathrm{mg}$ protein lysates were loaded to SDS-PAGE gel, and transferred to PVDF membranes (Millipore Corp., Billerica, MA, USA). The membrane was blocked in 5\% nonfat milk and incubated with diluted antibodies against ID2 (1:500; Invitrogen Life Technologies), followed by incubation with a horseradish peroxidase-conjugated secondary antibody (1:5,000; Santa Cruz Biotechnology, Inc., Santa Cruz, CA). $\beta$-actin was used as a control (1:1,000; Cell Signaling Technology, Inc., Danvers, MA, USA). 
Table I. Sequences of primers and siRNA used in this study.

\begin{tabular}{ll}
\hline Names & \multicolumn{1}{c}{ Sequences } \\
\hline qPCR primers & \\
H19-F & 5'-CGGACACAAAACCCTCTA-3' \\
H19-R & $5^{\prime}$-CTAGCTTCACCTTCCAGA-3' \\
GAPDH-F & $5^{\prime}$-GAAGGTGAAGGTCGGAGTC-3' \\
GAPDH-R & 5'-GAAGATGGTGATGGGATTTC-3' \\
ID2-F & 5'-CATCCCACTATTGTCAGC-3' \\
ID2-R & 5'-AATTCAGAAGCCTGCAAG-3' \\
siRNA & \\
H19 siRNA & \\
Plasmid construction & 5'-CCCGUCCCUUCUGAAUUUATT-3' \\
ID2-3'UTR reporter-F & \\
ID2-3'UTR reporter-R & \\
ID2-pcDNA4-F & CCGCTCGAGATAAGCGGTGTTCATGAT \\
ID2-pcDNA4-R & ATAAGAATGCGGCCGCTTTGACTTCAGACATGTTT \\
\hline
\end{tabular}

F, forward; R, reverse; qPCR, quantitative polymerase chain reaction; siRNA, small interfering RNA; ID2, inhibitor of DNA binding 2; 3'UTR, 3' untranslated region.

Dual luciferase reporter assay. Cotransfection was conducted in HEK-293T cells with $0.8 \mu \mathrm{g}$ plasmid and $50 \mu \mathrm{M}$ miRNA mimic. After transfected for $24 \mathrm{~h}$, the cells were harvested and treated according to instructions of Promega Dual-Luciferase ${ }^{\circledR}$ Reporter activity assay kit (Promega Corp., Fitchburg, WI, USA). The luciferase activity was then detected by a single photon detector. The relative luciferase activity was calculated as firefly luciferase activity value divided by Renilla reniformis luciferase activity. The experiment was repeated three times.

Statistical analysis. The expression differences between bone marrow samples of AML patients and health individuals were analyzed using paired samples t-test. Pearson's coefficient correlation was used for expression correlation assay. MTT assays were analyzed using ANOVA. The statistical analyses were performed using SPSS (Version 19.0; SPSS, Inc., Chicago, IL, USA). A two-sided $\mathrm{P}<0.05$ was considered to indicate a statistically significant difference.

\section{Results}

LncRNA H19 expression was upregulated in the bone marrow samples of AML-M2 patients. Many studies have shown that IncRNA H19 expression was upregulated in various tumorigenesis (9-16), which suggest that lncRNA H19 could serve as an oncogene in cancer. The expression level of lncRNA H19 in the bone marrow of AML-M2 patients (M2) was also higher (Fig. 1). It suggested that the aberrant expression of lncRNA H19 may be involved in the progression of AML.

Dalton et al have shown that HL-60 cell line was derived from a patient with FAB-M2 and not FAB-M3 (17). Therefore, HL-60 and KG-1a cell lines were chosen for further investigation in this study. The HL-60cell line was transfected with negative control or si-lncRNA H19. Three different siRNAs

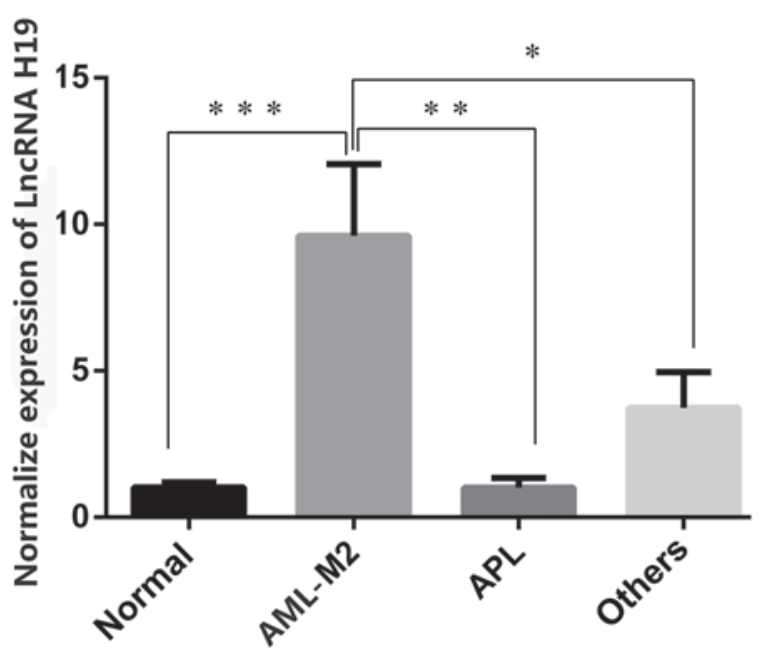

Figure 1. The expression of long non-coding RNA (lncRNA) H19 in bone marrows of acute myelocytic leukemia (AML) patients. Compared with healthy subjects (NORMAL), patients with acute promyelocytic leukemia (APL) and other types (OTHERS), relative expression of lncRNA H19 in AML-M2 (M2) was higher $\left({ }^{*} \mathrm{P}<0.05 ;{ }^{* *} \mathrm{P}<0.01 ;{ }^{* * *} \mathrm{P}<0.001\right)$.

were designed against H19 (named as si-H19a, si-H19b, si-H19c). Two of them can efficiently knockdown H19 expression, and eventually si-H19a was chosen for its best efficiency in the following experiments (Fig. 2A). The MTT assays and the soft agar colony formation assays showed that proliferation of HL-60 cell transfected with si-lncRNA H19 was also markedly decreased (Fig. 2B and C). Similar results were obtained with KG-1a cell line (Fig. 3).

Restored expression of ID2 partially reversed the effect of IncRNA H19 on AML cell proliferation. The possible mechanism involved in the regulation of AML cell proliferation by IncRNA H19 was unclear. It was proved that the 
A

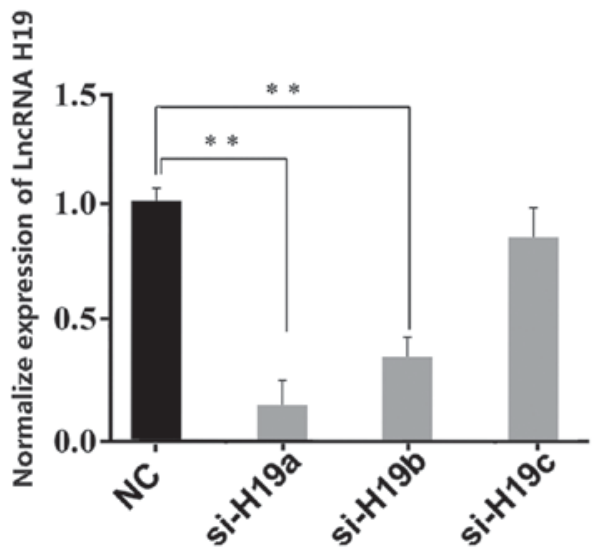

C

NC

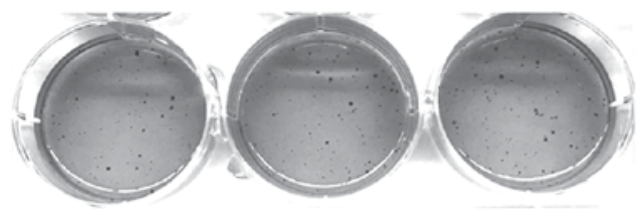

si-H19

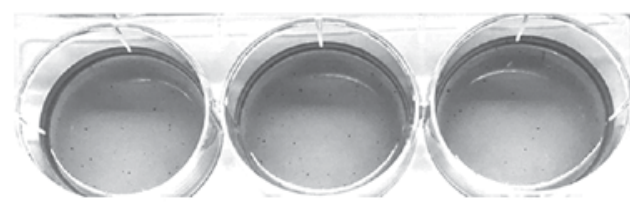

B

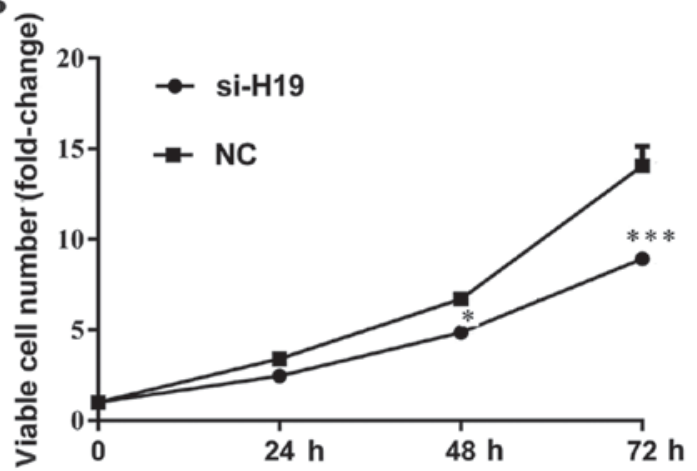

Figure 2. Knockdown of long non-coding RNA (IncRNA) H19 inhibited acute myelocytic leukemia cell proliferation. (A) si-lncRNA H19 could significantly reduce the expression of lncRNA H19. si-lncRNA H19 (si-H19) and negative control (NC) were transfected into HL-60 cell lines. LncRNA H19 expression was detected by qPCR. (B) Cell proliferation assay was performed by MTT assay. After transfected with si-H19 and NC for $72 \mathrm{~h}$, the rates of cell proliferation in the treatment of si-H19 were significantly slower than NC. (C) Cell proliferation assay was performed by soft agar colony formation assay. After transfected with si-H19 and $\mathrm{NC}$ for $72 \mathrm{~h}$, the number of colony formation in in the treatment of si-H19 was significantly slower than $\mathrm{NC}\left({ }^{*} \mathrm{P}<0.05 ;{ }^{* *} \mathrm{P}<0.01 ;{ }^{* * *} \mathrm{P}<0.001\right)$.
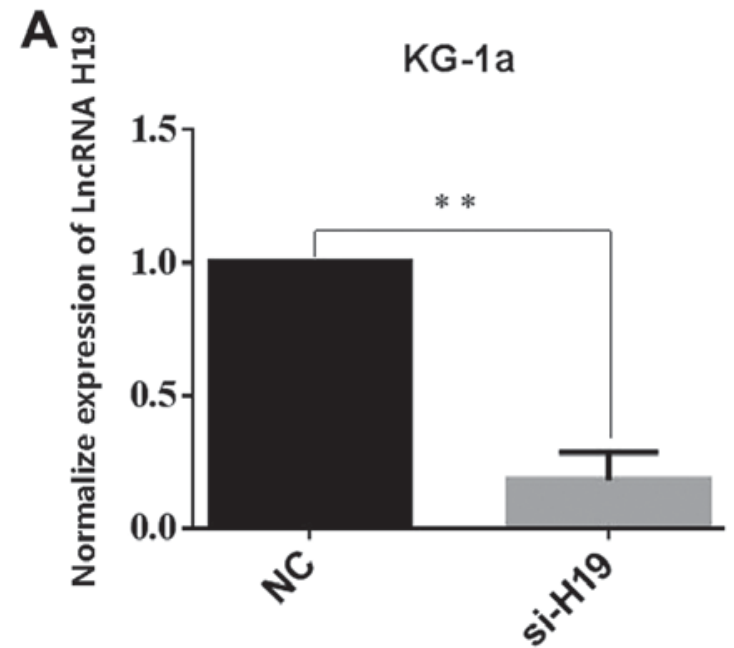

B

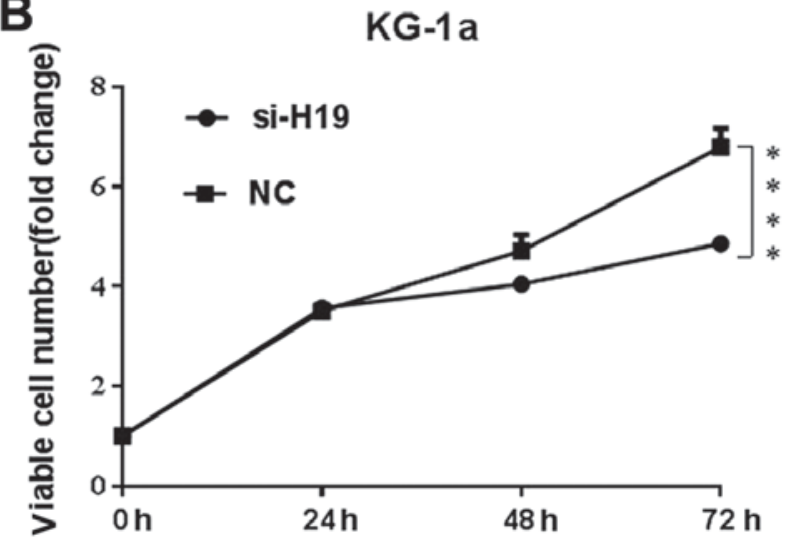

Figure 3. Knockdown of long non-coding RNA (lncRNA) H19 inhibited KG-1a cell proliferation. (A) Si-lncRNA H19 could significantly reduce the expression of lncRNA H19. Si-lncRNA H19 (si-H19) and negative control (NC) were transfected into KG-1a cell lines. LncRNA H19 expression was detected by qPCR. (B) Cell proliferation assay was performed by MTT assay. After transfected with si-H19 and NC for $72 \mathrm{~h}$, the rates of KG-1a cell proliferation in the treatment of si-H19 were significantly slower than NC. $\left({ }^{*} \mathrm{P}<0.05 ;{ }^{* *} \mathrm{P}<0.01\right)$.

expression of IncRNA H19 was related to the expression of ID2 in bladder cancer, which may promote bladder cell proliferation (18). Many studies have shown that expression of ID2 protein is increased in a large variety of human tumors, including bladder cancer, breast cancer, and squamous cell carcinoma (18-20). LncRNA H19 could serve as a competing endogenous RNA (ceRNA) by sponging hsa-miR-19a-3p in gastric cancer (21). Bioinformatics analysis indicated that the binding sites of hsa-miR-19a-3p and hsa-mir-19b-3p existed in the IncRNA H19 and 3'-untranslated region of ID2 


\section{A}

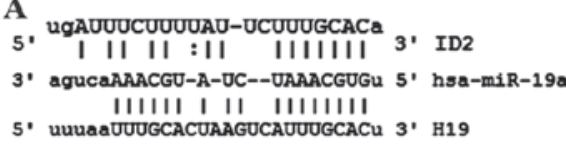

5. UgAUUUCUUUUAU-UCUUUGCACa 3, ID2

3' aguCAAAACGU-ACCUAAACGUGu 5 ' hsa-miR-19b

5. UCCCUUCUGAAUUUAAUUUGCACU 3. H19
B

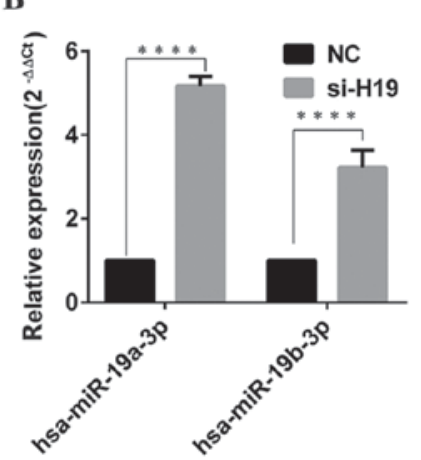

$\mathbf{E}$

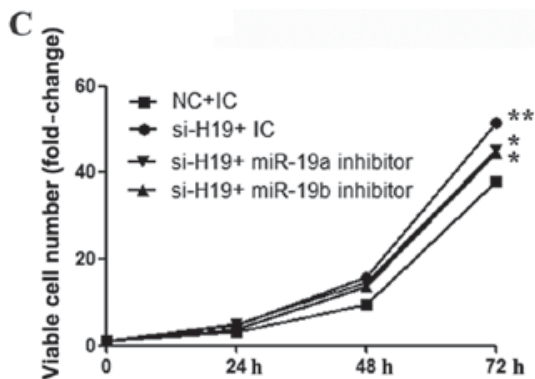

ID2
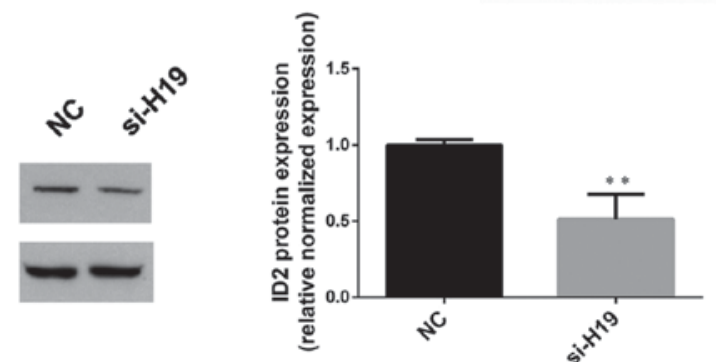

Figure 4. Knockdown of long non-coding RNA (lncRNA) H19 decreased inhibitor of DNA binding 2 (ID2) expression and increased hsa-miR-19a-3p and hsa-mir-19b-3p expression. (A) Seed match of hsa-miR-19a/b-3p, lncRNA H19 and ID2. (B) Hsa-miR-19a-3p expression in HL-60 cells transfected with negative control (NC) or si-lncRNA H19 (si-H19) was detected by qPCR. (C) Cell proliferation assay was performed by MTT assay. After transfected with si-H19, $\mathrm{miR}-19 \mathrm{a} / \mathrm{b}$ inhibitor and related controls for $72 \mathrm{~h}$, the rates of cell proliferation in the treatment of si-H19 were significantly restored by miR-19a/b inhibitor (D) Expression level of ID2 mRNA in HL-60 cells transfected with NC or si-H19 was detected by qPCR. (E) LncRNA H19 knockdown reduced the ID2 protein expression. GAPDH was used as internal loading control $\left.{ }^{* *} \mathrm{P}<0.01 ;{ }^{* * *} \mathrm{P}<0.001\right)$.

A

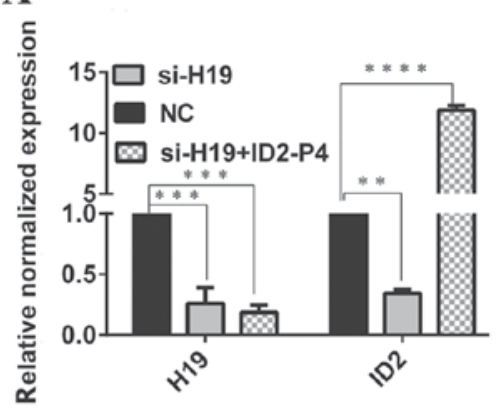

C

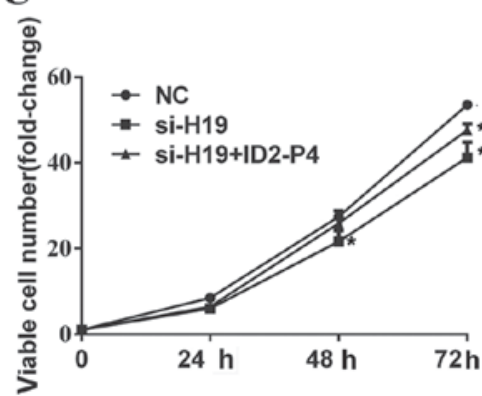

B

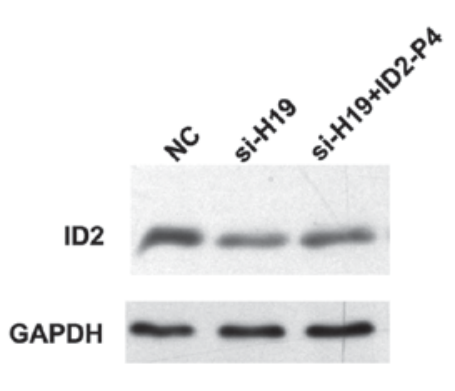

D

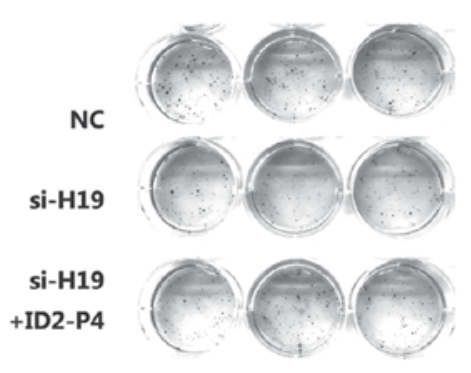

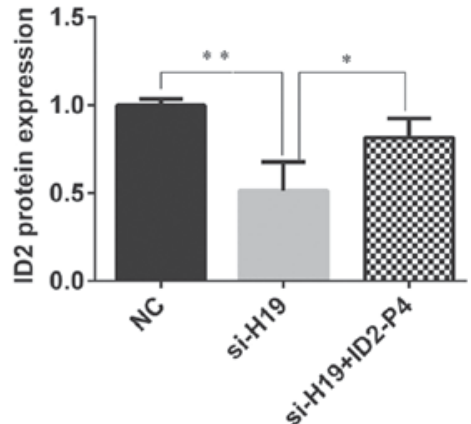

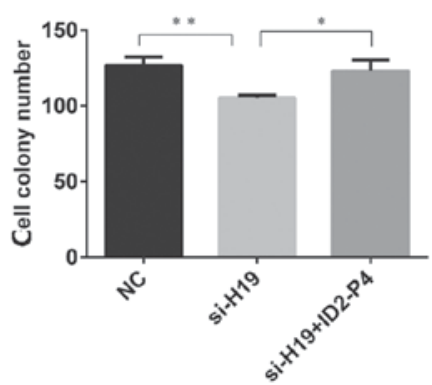

Figure 5. Restored inhibitor of DNA binding 2 (ID2) partially restored the effect of long non-coding RNA (lncRNA) H19 in AML cell proliferation. (A) LncRNA H19 and ID2 expression were detected by qPCR. (B) LncRNA H19 and ID2 expression were detected by western blotting. (C) MTT assay showed the effect of lncRNA H19 in cell proliferation partially reversed by restored ID2. (D) Colony formation assay showed the effect of lncRNA H19 in cell proliferation partially reversed by restored ID2 $\left({ }^{*} \mathrm{P}<0.05 ;{ }^{* *} \mathrm{P}<0.01 ;{ }^{* * *} \mathrm{P}<0.001\right)$.

(Fig. 4A). Hsa-miR-19a-3p and hsa-mir-19b-3p expressions were upregulated in HL-60 cells transfected with si-lncRNA H19 (Fig. 4B). Both inhibitors of hsa-miR-19a-3p and hsa-mir-19b-3p reversed the effect of lncRNA H19 on AML cell proliferation (Fig. 4C), while both mRNA and protein of ID2 was decreased (Fig. 4D and E). 
A

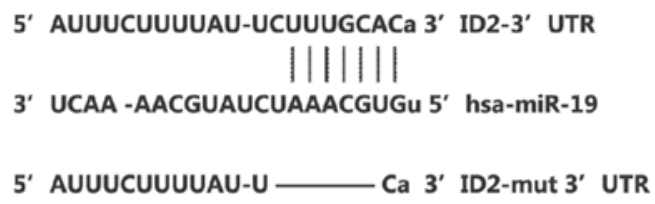

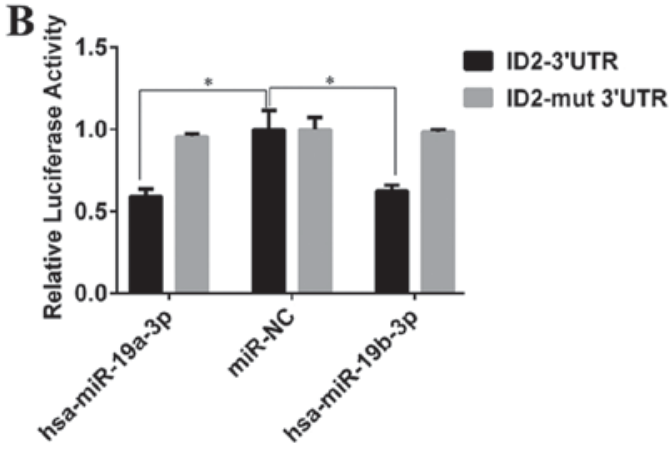

C

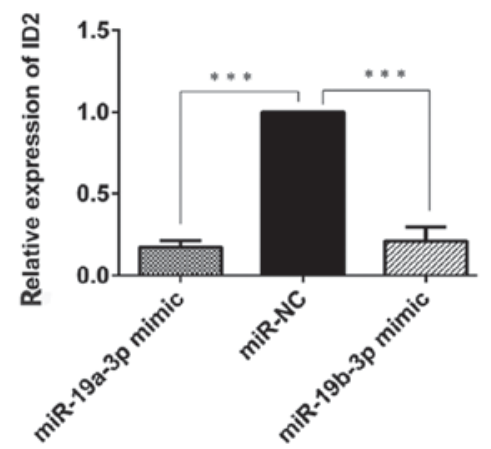

D

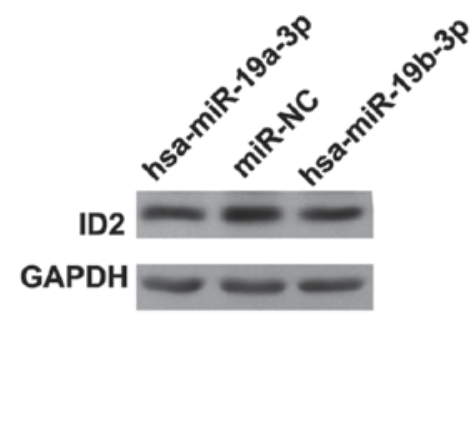

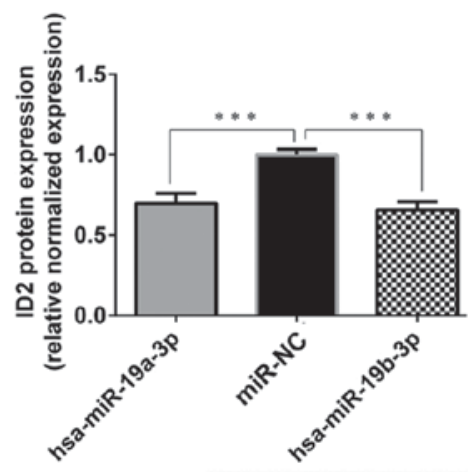

Figure 6. Inhibitor of DNA binding 2 (ID2) was a target of hsa-miR-19a-3p and hsa-miR-19b-3p. (A) Seed match of ID2 3' untranslated region (3'UTR) and hsa-miR-19a/b-3p. The 7 mer seed sequence in the 3'UTR of ID2 gene corresponding to hsa-miR-19a/b-3p binding site was marked, and the mutated-type fragments were generated by deletion of binding site. (B) Dual luciferase reporter assay showed that hsa-miR-19a-3p and hsa-miR-19b-3p mimics reduced relative luciferase activity of ID2-3'UTR rather than ID2-mut 3'UTR plasmid. (C) ID2 mRNA and protein expression were detected by qPCR. (D) hsa-miR-19a-3p and hsa-miR-19b-3p mimics significantly decrease the ID2 protein expression $\left({ }^{*} \mathrm{P}<0.05 ;{ }^{* * *} \mathrm{P}<0.001\right)$.

To confirm that IncRNA H19 may promote AML cell proliferation by regulating ID2 protein expression, knockdown of IncRNA H19 and overexpression of ID2 were performed in HL-60 cells simultaneously. As a result, restored expression of ID2 reversed the effect of IncRNA H19 on AML cell proliferation (Fig. 5). It was suggested that lncRNA H19 may increase AML cell proliferation by regulating ID2.

Hsa-miR-19a-3p and hsa-mir-19b-3p inhibited ID2 expression. One potential binding site for hsa-miR-19a-3p and hsa-miR-19b-3p was located in the ID2-3'UTR (Fig. 6A). Cotransfection with ID2-3'UTR and hsa-miR-19a/b-3p mimic caused a significant decrease in the luciferase activity compared with negative control. In contrast, the activity of the reporter construct containing the mutant site was not changed (Fig. 6B). Furthermore, ID2 mRNA and protein expression were reduced in transfected HL-60 cells (Fig. 6C and D). These results suggested that hsa-miR-19a-3p and hsa-miR-19b-3p exerted an inhibitory effect on ID2 expression via binding 3'UTR of ID2.

\section{Discussion}

ID2 is an important regulator in cell proliferation and differentiation, which plays critical roles in development and cancer. It suggests that ID2 serves as an oncogene in cancer. There was high ID2 expression in immature granulocyte, and the level of ID2 protein expression in acute leukemia blast cells was significantly higher (22). Luo et al have also mentioned that the expression of lncRNA H19 was positively correlated with that of ID2 in bladder cancer tissues. Furthermore, ID2 partially reversed the effect of lncRNA H19 on cell proliferation (18). In this paper, we also found that the expression of ID2 was downregulated in AML cells after lncRNA H19 was knockdown. Taken together, it suggests that lncRNA H19 might play a role in AML cell proliferation through regulating ID2 expression, but the specific mechanism is still needed to be elucidated.

MicroRNAs (miRNA) are small noncoding RNA fragments approximately 22 nucleotides long, which are involved in post-transcriptional regulation of gene expression. MiRNAs can bind transcripts through sequence complementarity to either promote mRNA degradation or prevent mRNA translation. Salmena et al have proposed a ceRNA hypothesis (23). Protein-coding and ncRNAs, which share one or more miRNA response elements (MREs), may compete for binding to miRNAs and regulate expression of each other. LncRNA H19 was considered as a ceRNA of miR-138, miR-200a, let-7, miR-106a, miR-141 and miR-22 (14,24,25). In this research, according to bioinformative analysis, there were hsa-miR-19a/b-3p binding sites in lncRNA H19 and ID2, which indicated that IncRNA H19 regulate ID2 expression by binding hsa-miR-19a/b-3p.

In conclusion, we found that the expression of lncRNA H19 in bone marrow samples of AML-M2 patients is higher. Knockdown of lncRNA H19 resulted in upregulated expression of hsa-miR-19a/b-3p. LncRNA H19 inhibited ID2 mRNA and protein expression, which promoted AML cell proliferation. 


\section{Acknowledgements}

This study was supported by National Natural Scientific Foundation of China (nos. 81402600 and 81670175), the Science and Technology Development Fund Project of Shenzhen (nos. JCYJ20150403110829622 and JCYJ20160427185541943).

\section{References}

1. Ferrara F and Schiffer CA: Acute myeloid leukaemia in adults. Lancet 381: 484-495, 2013.

2. Kapranov P, Cheng J, Dike S, Nix DA, Duttagupta R, Willingham AT, Stadler PF, Hertel J, Hackermüller J, Hofacker IL, et al: RNA maps reveal new RNA classes and a possible function for pervasive transcription. Science 316: 1484-1488, 2007.

3. Gibb EA, Brown CJ and Lam WL: The functional role of long non-coding RNA in human carcinomas. Mol Cancer 10: 38, 2011.

4. Perez DS, Hoage TR, Pritchett JR, Ducharme-Smith AL, Halling ML, Ganapathiraju SC, Streng PS and Smith DI: Long, abundantly expressed non-coding transcripts are altered in cancer. Hum Mol Genet 17: 642-655, 2008.

5. Louro R, Smirnova AS and Verjovski-Almeida S: Long intronic noncoding RNA transcription: Expression noise or expression choice? Genomics 93: 291-298, 2009.

6. Khalil AM, Guttman M, Huarte M, Garber M, Raj A, Rivea Morales D, Thomas K, Presser A, Bernstein BE, van Oudenaarden A, et al: Many human large intergenic noncoding RNAs associate with chromatin-modifying complexes and affect gene expression. Proc Natl Acad Sci USA 106: 11667-11672, 2009.

7. Li L, Feng T, Lian Y, Zhang G, Garen A and Song X: Role of human noncoding RNAs in the control of tumorigenesis. Proc Natl Acad Sci USA 106: 12956-12961, 2009.

8. Verhaegh GW, Verkleij L, Vermeulen SH, den Heijer M, Witjes JA and Kiemeney LA: Polymorphisms in the H19 gene and the risk of bladder cancer. Eur Urol 54: 1118-1126, 2008.

9. Zhu Z, Song L, He J, Sun Y, Liu X and Zou X: Ectopic expressed long non-coding RNA H19 contributes to malignant cell behavior of ovarian cancer. Int J Clin Exp Pathol 8: 10082-10091, 2015.

10. Berteaux N, Lottin S, Monté D, Pinte S, Quatannens B, Coll J, Hondermarck H, Curgy JJ, Dugimont T and Adriaenssens E: H19 mRNA-like noncoding RNA promotes breast cancer cell proliferation through positive control by E2F1. J Biol Chem 280: 29625-29636, 2005.

11. Wu T, Qu L, He G, Tian L, Li L, Zhou H, Jin Q, Ren J, Wang Y, Wang $\mathrm{J}$, et al: Regulation of laryngeal squamous cell cancer progression by the lncRNA H19/miR-148a-3p/DNMT1 axis. Oncotarget 7: 11553-11566, 2016.
12. Wang L, Cai Y, Zhao X, Zhang J, Liu J, Zhen H, Wang T, Tang X, Liu Y and Wang J: Down-regulated long non-coding RNA H19 inhibits carcinogenesis of renal cell carcinoma. Neoplasma 62: 412-418, 2015.

13. Zhou X, Yin C, Dang Y, Ye F and Zhang G: Identification of the long non-coding RNA H19 in plasma as a novel biomarker for diagnosis of gastric cancer. Sci Rep 5: 11516, 2015.

14. Zhou X, Ye F, Yin C, Zhuang Y, Yue G and Zhang G: The interaction between MiR-141 and lncRNA-H19 in regulating cell proliferation and migration in gastric cancer. Cell Physiol Biochem 36: 1440-1452, 2015.

15. Zhang E, Li W, Yin D, De W, Zhu L, Sun S and Han L: c-Myc-regulated long non-coding RNA H19 indicates a poor prognosis and affects cell proliferation in non-small-cell lung cancer. Tumour Biol 37: 4007-4015, 2016.

16. Luo M, Li Z, Wang W, Zeng Y, Liu Z and Qiu J: Long non-coding RNA H19 increases bladder cancer metastasis by associating with EZH2 and inhibiting E-cadherin expression. Cancer Lett 333: 213-221, 2013

17. Dalton WT Jr, Ahearn MJ, McCredie KB, Freireich EJ, Stass SA and Trujillo JM: HL-60 cell line was derived from a patient with FAB-M2 and not FAB-M3. Blood 71: 242-247, 1988.

18. Luo M, Li Z, Wang W, Zeng Y, Liu Z and Qiu J: Upregulated H19 contributes to bladder cancer cell proliferation by regulating ID2 expression. FEBS J 280: 1709-1716, 2013

19. Li K, Yao L, Chen L, Cao ZG, Yu SJ, Kuang XY, Hu X and Shao ZM: ID2 predicts poor prognosis in breast cancer, especially in triple-negative breast cancer, and inhibits E-cadherin expression. Onco Targets Ther 7: 1083-1094, 2014

20. Wang C, Chen Q, Hamajima Y, Sun W, Zheng YQ, Hu XH, Ondrey FG and Lin JZ: Id2 regulates the proliferation of squamous cell carcinoma in vitro via the $\mathrm{NF}-\kappa \mathrm{B} / \mathrm{Cyclin} \mathrm{D} 1$ pathway. Chin J Cancer 31: 430-439, 2012

21. Xia T, Liao Q, Jiang X, Shao Y, Xiao B, Xi Y and Guo J: Long noncoding RNA associated-competing endogenous RNAs in gastric cancer. Sci Rep 4: 6088, 2014.

22. May AM, Frey AV, Bogatyreva L, Benkisser-Petersen M, Hauschke D, Lübbert M, Wässch R, Werner M, Hasskarl J and Lassmann S: ID2 and ID3 protein expression mirrors granulopoietic maturation and discriminates between acute leukemia subtypes. Histochem Cell Biol 141: 431-440, 2014.

23. Salmena L, Poliseno L, Tay Y, Kats L and Pandolfi PP: A ceRNA hypothesis: The Rosetta Stone of a hidden RNA language? Cell 146: 353-358, 2011

24. Liang WC, Fu WM, Wong CW, Wang Y, Wang WM, Hu GX, Zhang L, Xiao LJ, Wan DC, Zhang JF and Waye MM: The lncRNA H19 promotes epithelial to mesenchymal transition by functioning as miRNA sponges in colorectal cancer. Oncotarget 6: 22513-22525, 2015.

25. Liang WC, Fu WM, Wang YB, Sun YX, Xu LL, Wong CW, Chan KM, Li G, Waye MM and Zhang JF: H19 activates Wnt signaling and promotes osteoblast differentiation by functioning as a competing endogenous RNA. Sci Rep 6: 20121, 2016. 